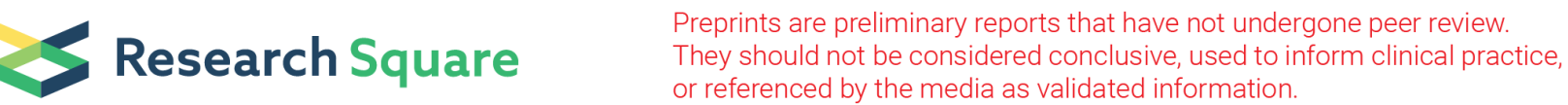

\section{Embryonic Stem Cell-derived Mesenchymal Stem Cells Alleviate Skeletal Muscle Injury Induced by Acute Compartment Syndrome}

Xiangkang Jiang ( $\sim$ jxk1996@zju.edu.cn )

Zhejiang University

Jingyuan Yang

Zhejiang University

Fei Liu

Zhejiang University

Jiefeng Xu

Zhejiang University

Mao Zhang

Zhejiang University

\section{Research Article}

Keywords: Acute compartment syndrome, Skeletal muscle injury, Embryonic stem cells, Mesenchymal stem cells, M2 macrophage

Posted Date: February 15th, 2022

DOI: https://doi.org/10.21203/rs.3.rs-1338955/v1

License: (c) (i) This work is licensed under a Creative Commons Attribution 4.0 International License.

Read Full License 


\section{Abstract \\ Background}

Acute compartment syndrome (ACS), a well-known complication of musculoskeletal injury, results in muscle necrosis and cell death. Embryonic stem cell-derived mesenchymal stem cells (ES-MSCs) have been shown to be a promising therapy for ACS. However, their effectiveness and potentially protective mechanism remain unknown. The present study was designed to investigate the efficacy and underlying mechanism of ES-MSCs in ACS-induced skeletal muscle injury.

\section{Method}

A total of 120 male Sprague-Dawley (SD) rats underwent 2 hours of intracompartmental pressure elevation by saline infusion into the anterior compartment of the left hindlimb to establish the ACS model. ES-MSCs were differentiated from the human embryonic stem cell (ES) line H9. A dose of $1.2 \times 10^{6}$ of ES-MSCs was intravenously injected during fasciotomy. Post-ACS assessments included skeletal edema index, serum indicators, histological analysis, apoptosis, fibrosis, regeneration, and functional recovery of skeletal muscle. Then, fluorescence microscopy was used to observe the distribution of labeled ES-MSCs in vivo, and western blotting and immunofluorescence analyses were performed to examine M2 macrophage infiltration in skeletal muscle. Finally, we used liposomal clodronate to deplete macrophages and reassess skeletal muscle injury in response to ES-MSC therapy.

\section{Result}

ES-MSCs significantly reduced systemic inflammatory responses, ACS-induced skeletal muscle edema and cell apoptosis. In addition, ES-MSCs inhibited skeletal muscle fibrosis and increased regeneration and functional recovery of skeletal muscle after ACS. The beneficial effects of ES-MSCs on ACS-induced skeletal muscle injury were accompanied by an increase in CD206-positive M2 macrophage polarization. After depleting macrophages with liposomal clodronate, the beneficial effects of ES-MSCs were abolished.

\section{Conclusion}

Our findings suggest that embryonic stem cell-derived mesenchymal stem cells infusion could effectively alleviate ACS-induced skeletal muscle injury, in which the protective effects were related to M2 macrophage polarization.

\section{Background}


Acute compartment syndrome (ACS), characterized by increasing osseofascial pressure with a severe skeletal muscle injury, is a surgical emergency in clinical practice [1]. Fasciotomy, to fully decompress all compartments, remains the only gold standard treatment for ACS [2, 3]. Despite reperfusion by fasciotomy, paradoxical skeletal muscle injury, which is referred to as ischemia-reperfusion injury (IRI), accompanies ACS. IRI triggers further skeletal muscle damage, which can lead to hypovolemia, rhabdomyolysis, electrolyte and acid-base imbalances, renal failure, and sometimes death $[4,5]$. A previous article [6] reported that $44 \%$ of ACS patients have long-term limb function defects after fasciotomy. Therefore, it is urgent to develop novel therapeutic methods for reperfusion-associated skeletal injury in ACS.

Mesenchymal stem cells (MSCs), derived from bone marrow, placenta, adipose, or other tissues, have been proven to be a promising therapy against IRI-related skeletal muscle injury [7-10]. MSC therapy can reduce neutrophil infiltration in injured skeletal muscle and cell apoptosis caused by inflammation [8] and promote skeletal muscle regeneration and functional recovery after injury $[7,10]$. However, MSC populations possess heterogeneous biological properties, which could affect their therapeutic efficacy in diseases [11]. Moreover, the numbers of harvested MSCs and their in vitro expansion were a challenge $[12,13]$. The successful isolation and culture of embryonic stem cells (ES) in vitro [14], which are capable of unlimited self-renewal and differentiation into all cell lineages, opened a new avenue for MSC derivation [15-17].

ES-derived MSCs (ES-MSCs) are characterized by a uniform phenotype, relatively stronger proliferation capacity, and stable immunomodulatory properties, and these cells are suitable for large-scale culture. Compared with MSCs from other sources, ES-MSCs achieve better efficacy in diseases $[18,19]$. These properties and evidence indicate that ES-MSCs may serve as better candidates for off-the-shelf cell products in future clinical applications than currently available therapies. The efficacy of ES-MSC infusion in ACS-induced skeletal muscle injury has not been reported, and its potentially protective mechanism requires further investigations.

In this study, we generated MSCs with the same phenotype from embryonic stem cells (ES-MSCs) and investigated their therapeutic potential in ACS rats model. In addition, the molecular basis for their therapeutic effect will be discussed.

\section{Material And Methods}

\section{Animals and ethical statement}

All animal experiments were approved by the Institutional Ethics Committee of the Second Affiliated Hospital of Zhejiang University School of Medicine. Male Sprague-Dawley (SD) rats (two months old, $250 \pm 10 \mathrm{~g}$ ) were purchased from Slac Laboratory Animal Co., Ltd. (Shanghai, China) and were housed in a temperature-controlled $\left(22 \pm 1^{\circ} \mathrm{C}\right)$ and humidity-controlled $(60 \pm 5 \%)$ room under a 12-hour light-dark cycle. The animals had free access to food and water. 


\section{ES-MSCs preparation and identification}

ES-MSCs were differentiated from ES using a two-step process. In brief, H9-ES colonies (YuanSheng Biotech Corporation, Hangzhou, China) were dissociated into small clumps after 3 minutes of incubation with TrypLE Express and then transferred to ultralow-attachment plates in E8 media (Gibco, Grand Island, NY, USA). After 7 days, embryoid bodies (EBs) were harvested and plated in MSC induction medium composed of Dulbecco's modified Eagle's medium (high glucose), $10 \%$ fetal bovine serum, and $1 \mathrm{mM} \mathrm{L-}$ glutamine. After $2 \mathrm{w}$, the EB outgrowths were subcultured using TrypLE Express. These cells were ESMSCs and were designated passage 0 (P0). The differentiated ES-MSCs attained a homogenous population of spindle-shaped cells. Passage 4 (P4) ES-MSCs were used in the following animal experiment.

In this study, phenotypic identification of MSCs was performed using flow cytometry (BD Biosciences, USA) with the following monoclonal antibodies: anti-CD73-PE-Cy7, anti-CD90-APC, anti-CD105-PE, antiCD14-APC, anti-CD34-PE, anti-CD45-fluorescein isothiocyanate (FITC), CD79a-PE, and HLA-DR-PE. All antibodies were purchased from BD Pharmingen (China). After antibody labeling, data were acquired using an Agilent NovoCyte and analyzed using NovoExpress.

\section{Establishment of the ACS animal model}

The ACS model was established by infusing isotonic normal saline as previously described [20, 21]. In brief, the rats were anesthetized by intraperitoneal administration of $3 \%$ pentobarbital sodium. Once the animals were anesthetized, a 24-gauge angiocatheter was inserted into the anterior compartment of the left hindlimb in the experimental group to elevate compartment pressure. The intracompartmental pressure was increased to $80 \mathrm{mmHg}$ and maintained between $80 \pm 10 \mathrm{mmHg}$ for 2 hours, and a singleincision fasciotomy was performed to decompress the compartment at the end of the experiment. Sham animals underwent all procedures, but the compartment pressure was maintained at the baseline level (0 $\mathrm{mmHg})$. Rats were sacrificed by an intra-arterial overdose $(1.5 \mathrm{ml})$ of $3 \%$ pentobarbital sodium, and blood, lung, and tibialis anterior (TA) muscle samples were harvested for further processing.

\section{Experimental design}

This study consisted of three separate parts (Fig. 2). A total of 120 rats were used in this study.

\section{Part 1}

To investigate the therapeutic effects of ES-MSCs on ACS-induced skeletal muscle injury, rats were randomly divided into four groups: the Sham+PBS, Sham+MSCs, ACS+PBS, and ACS+MSCse groups at different time points ( 1 day, 3 days, and 7 days). After fasciotomy, ES-MSCs $\left(1.2 \times 10^{6}\right.$ in $0.5 \mathrm{ml}$ of PBS) or an equal volume of PBS was administered to the rats by the dorsal penis vein. The muscle edema index and serum creatine kinase (CK) level were measured at 1 day, 3 days, and 7 days after ACS. Moreover, enzyme-linked immunosorbent assay (ELISA) and hematoxylin and eosin (H\&E) staining were performed 1 day after ACS. Terminal dUTP nick end labeling (TUNEL) staining and western blotting were performed 
3 days after ACS. Additionally, rat ethology was tested 7 days after model induction, and H\&E and Masson trichrome staining of the TA muscle was performed 7 days after ACS.

\section{Part 2}

To clarify whether M2 macrophage induction was involved in ES-MSC therapy, rats were randomly assigned to two groups: the ACS+PBS and ACS+MSCs groups at different time points (0 hours, 1 day, 3 days, 5 days, and 7 days). Cells were labeled with Paul Karl Horan fluorescent dye (PKH26) before being intravenously injected to track the distribution of ES-MSCs in vivo. Western blotting and immunofluorescence analysis was performed at 0 days, 1 day, 3 days, 5 days, and 7 days after ACS. Moreover, quantitative real-time PCR was performed 3 days after ACS.

Part 3

To identify the role of M2 macrophages in ES-MSC therapy, macrophages were depleted in rats by intravenous injection of liposomal clodronate (LC). Sham rats were injected with the same amount of liposomal vehicle (LV). Serum CK analysis, H\&E staining, quantitative real-time PCR, immunohistochemistry, and western blotting were performed 3 days after macrophage depletion and ESMSC therapy.

\section{ES-MSCs labeling}

For ES-MSC labeling, we incubated the cells with PKH26 $(10 \mu \mathrm{M})$ (Sigma, St Louis, MO, USA) at $37^{\circ} \mathrm{C}$ for 5 min. After the cells were washed with PBS three times, ES-MSCs were diluted to a concentration of $2.4 \times$ $10^{6} \mathrm{cell} / \mathrm{ml}$ in PBS for injection.

\section{Macrophages depletion}

For the macrophage depletion studies, rats were intravenously injected with $1 \mathrm{ml}$ of $(5 \mathrm{mg} / \mathrm{ml})$ LC (Vrije Universiteit, Amsterdam, Netherlands) 1 day prior to and 1 day following ACS injury as previously described [22]. Rats injected with LV (Vrije Universiteit) were used as the control.

\section{Blood sampling and serum analysis}

Blood samples were collected from the abdominal aorta, and serum was obtained by centrifugation. Serum CK levels were measured using a commercial CK kit (Jiancheng Bioengineering Institute, Nanjing, China). The concentrations of tumor necrosis factor-alpha (TNF-a), interleukin 6 (IL-6) and interleukin 10 (IL-10) were measured using ELISA kits (Elabscience, Wuhan, China). The optical density (OD) was measured at $450 \mathrm{~nm}$ using a microplate reader (Thermo Fisher Scientific, MA, USA).

\section{Muscle edema index}

The left and right TA muscles were harvested and weighed immediately, and the left/right TA muscle weight ratio was calculated to determine the muscle edema index.

\section{Rat ethology}


The hanging grid test and grip strength test were used to assess skeletal muscle function. The hanging grid test was performed as described previously for mice [23]. The rats were placed individually at the center of a wire mesh screen ( $2 \mathrm{~mm}$ wire thickness). The screen was suspended $50 \mathrm{~cm}$ above a plastic cage filled with sawdust bedding, and the grid was inverted with the head declining first. The hanging duration was recorded in three independent trials conducted at least 20 minutes apart. The data from all three trials were averaged.

The grip strength test was performed using a grip strength meter (Handpi HP-5N, Shenzhen, China) as previously described [24]. The rats were held by the tail, grasped a grid with their paws, and were gently pulled by the tail until they released their grip. The forces of three trials were recorded and averaged.

\section{Histological analysis}

The TA muscles were fixed in 10\% formalin for 24 hours and underwent routine dehydration and paraffin embedding. Tissue sections $(4 \mu \mathrm{m})$ were stained with $\mathrm{H} \& \mathrm{E}$ or Masson trichrome and examined under a light microscope (Leica, Germany).

H\&E staining was performed to evaluate the pathological damage to skeletal muscle at 1 day and skeletal muscle regeneration at 7 days after ACS. The histological damage score was determined using 5 random fields selected from each sample at an objective magnification of $\times 20$ as previously described [25], and the scoring was as follows: disorganization and degeneration of the muscle fibers (0: normal, 1 : mild, 2: moderate, 3: severe); and inflammatory cell infiltration (0: normal, 1: mild, 2: moderate, 3: severe). Regenerative myofibers were identified as those containing central nuclei [26]. Under an objective magnification of 20x, five random fields were selected from each sample and used to quantify the total number of regenerative myofibers. To measure the diameters of regenerative myofibers, the minor axis diameters of the regenerative myofibers were measured in each TA muscle as previously described [27].

Masson trichrome staining was performed to measure fibrosis 7 days after ACS. The fibrotic area of skeletal muscle was quantitated using 5 random fields selected from each sample at an objective magnification of $\times 20$. Image $J$ software was used to calculate the percentage of the fibrotic area.

Skeletal muscle fiber injury in Part 3 was evaluated 3 days after macrophage depletion and ES-MSC therapy. The injury score was determined based on a protocol established by McCormack et al. [28]. Four random fields of each $\mathrm{H} \& \mathrm{E}-$ stained section at an objective magnification of 20x were examined, and based on the proportion of injured cells (defined by ragged cellular edges, vacuolation, lymphocyte infiltration, or rhabdomyolysis), a numerical value between 0 and 10 was determined.

\section{TUNEL and dystrophin staining analysis.}

Apoptotic nuclei in skeletal muscle were examined using double-fluorescent labeling of TUNEL and dystrophin. TUNEL staining was performed according to the manufacturer's protocol (Roche Inc., Basel, Switzerland). After TUNEL labeling, tissue sections were incubated with a rabbit anti-dystrophin monoclonal antibody (1:200, Cat. 12715-1-AP, Proteintech) followed by an anti-rabbit IgG cyanin 3 (Cy3) 
(1:200, Cat. SA00009-2, Proteintech). Sections were incubated with DAPI (Meilunbio, Dalian, China) to stain nuclei and examined under a fluorescence microscope (Olympus, Tokyo, Japan). Photomicrographs were merged and saved by Image-Pro Plus software (Olympus). The numbers of TUNEL- and DAPIpositive nuclei were counted and only labeled nuclei that colocalized with dystrophin staining were counted. The data are expressed as the TUNEL index, which was calculated by counting the number of TUNEL-positive nuclei divided by the total number of nuclei. The TUNEL index for each muscle was calculated from five random, nonoverlapping fields at an objective magnification of 40x.

\section{Immunofluorescence staining}

Immunofluorescence staining was conducted as previously described [29] to detect M2 macrophage infiltration in skeletal muscle. Skeletal muscle sections were subjected to antigen retrieval, and the sections were blocked and labeled overnight at $4^{\circ} \mathrm{C}$ with rabbit anti-CD68 (1:200, Cat. sc-20060, Santa Cruz) and mouse anti-CD206 (1:200, Cat. sc-58986, Santa Cruz). After the sections were washed in PBS three times, FITC- or Cy3-labeled secondary antibodies (1:200, Proteintech) were used for the final immunostaining. Sections that were not incubated with primary antibodies were used as negative controls. The sections were incubated with DAPI (Meilunbio) to stain nuclei and examined under a fluorescence microscope (Olympus).

\section{Western blotting}

Western blotting was used to determine protein expression as previously described [30]. TA muscle tissue was lysed in RIPA lysis buffer (Biosharp, Hefei, China). After centrifugation, soluble proteins were quantified with a BCA kit (Biosharp) and separated by sodium dodecyl sulfate-polyacrylamide gel electrophoresis. The proteins were electrophoresed until sufficiently separated and then transferred to polyvinylidene difluoride (PVDF) membranes. The PVDF membranes were blocked with $5 \%$ nonfat dry milk in Tris-buffered saline, and then the PVDF membranes were incubated with the following primary antibodies: rabbit anti-tubulin (1:5000, Cat. \#5335S, CST), rabbit anti-cleaved caspase3 (1:1000, Cat. \#9664S, CST), rabbit anti-caspase3 (1:1000, Cat. \#9662S, CST), rabbit anti-Bax (1:1000, Cat. \#14796S, CST), rabbit anti-Bcl-2 (1:1000, Cat. \#3498S, CST), mouse anti-CD206 (1:1000, Santa Cruz), and rabbit anti-CD68 (1:1000, Santa Cruz) at $4^{\circ} \mathrm{C}$ overnight. Then, the PVDF membranes were incubated with corresponding horseradish peroxidase (HRP)-conjugated IgG antibodies at room temperature for 2 hours. Bands were visualized using an ECL kit (Millipore, Billerica, MA, USA). The band densities were quantified with ImageJ software (NIH).

\section{Quantitative real-time PCR}

RNA was extracted and analyzed using a previously described method [27]. Total RNA was obtained with TRIzol reagent (Invitrogen, MA. USA) and quantified by a Nanodrop spectrophotometer (Thermo Fisher). RNA was then reverse transcribed by the PrimeScript RT reagent kit (Yeason, Shanghai, China). Then, quantitative real-time PCR was performed with SYBR Mixture (Yeason), specific rat primers and CDNA using the Mx3000P real-time PCR system (Agilent Technologies, USA). $\beta$-Actin was used as the internal reference. The sequences of primers were used as follows: rat $\beta$-Actin forward: 5 '- 
TGTCACCAACTGGGACGATA-3', reverse: 5'-GGGGTGTTGAAGGTCTCAAA-3'; rat TNF-a forward: 5'ATGGGCTCCCTCTCATCAGTTCC-3', reverse: 5'-GCTCCTCCGCTTGGTGGTTTG-3'; rat IL-6 forward: 5'ACTTCCAGCCAGTTGCCTTCTTG-3', reverse: 5'-TGGTCTGTTGTGGGTGGTATCCTC-3'; rat IL-10 forward: 5'CTGCTCTTACTGGCTGGAGTGAAG-3', reverse: 5'-TGGGTCTGGCTGACTGGGAAG-3';

\section{Immunohistochemistry}

Immunohistochemistry was performed as previously described [31]. Skeletal muscle sections were subjected to antigen retrieval, and the sections were blocked and labeled overnight at $4^{\circ} \mathrm{C}$ with rabbit antiCD68 (1:200, Santa Cruz). After being incubated with HRP-conjugated secondary antibodies (Boster, Wuhan. China), the sections were treated with an avidin-biotin-peroxidase conjugate (Boster). The reaction was visualized using diaminobenzidine (DAB) substrate chromogen solution (Vectorlabs, CA, USA) after the tissue was counterstained with hematoxylin.

\section{Statistical analysis}

The data are expressed as the means \pm standard deviation (SD). One-way analysis of variance (ANOVA) and repeated-measures ANOVA were used for multivariate data analyses. Student's t-tests were used for post hoc statistical analyses. All statistical analyses were performed by SPSS (version 22.0), and a value of $P \leq 0.05$ was considered to indicate a statistically significant difference.

\section{Results}

\section{Generation and identification of ES-MSCs}

Differentiated ES-MSCs developed a homogenous population of spindle-shaped cells. P4 ES-MSCs were used in the animal experiment. ES-MSC morphology at P0 and P4 is shown in Fig. 1a.

The results of ES-MSC identification are shown in Fig. 1b, and the percentages of CD73-, CD90-, and CD105-positive cells were $99.84 \%, 99.89 \%$, and $96.57 \%$, respectively, while ES-MSCs were negative for CD14, CD34, CD45, CD79a, and HLA-DR (all <0.5\%).

\section{ES-MSCs reduce the muscle edema index and serum CK levels after ACS}

The left/right TA muscle weight ratio was used as an index of edema, and the muscle edema index was similar among the sham groups. ACS injury resulted in a notably increase in the muscle edema index, and ES-MSCs effectively reduced the muscle edema index 1 day after ACS (Fig. 3a). A similar trend was observed regarding serum CK levels (Fig. 3b). However, in our study, after 3 days, the muscle edema index and serum CK levels gradually returned to normal, and there were no significant differences between the ACS groups and sham groups. 


\section{ES-MSCs alleviate systemic inflammation and histopathological alterations in skeletal muscle after ACS}

To examine the effect of ES-MSCs on systemic inflammation and ACS-induced skeletal muscle injury, ELISA and histologic examination were performed. The ELISA results showed that ES-MSCs significantly decreased serum levels of the proinflammatory cytokines TNF- $a$ and IL- 6 and increased the antiinflammatory cytokine IL-10 (Fig. 4a-c). Severely disorganized and degenerated muscle fibers and inflammatory cell infiltration were observed in ACS rats (Fig. 4g). Infusion with ES-MSCs significantly reduced skeletal muscle fiber disorganization and inflammatory cell infiltration (Fig. 4h) and lowered the histological damage score (Fig. 4d).

\section{ES-MSCs decrease skeletal muscle apoptosis after ACS}

Apoptotic cells were examined using the TUNEL assay. Three days after ACS, rats in the ACS group exhibited a significant increase in the skeletal muscle apoptotic index, and ES-MSCs suppressed ACSinduced apoptosis in skeletal muscle cells (Fig. 5a). In addition, western blotting was used to determine the expression of apoptosis-related proteins in skeletal muscle, and the data suggested that the protein levels of cleaved caspase-3, Bax, and Caspase-3 were significantly increased, while the protein level of Bcl-2 was decreased in the ACS group compared to the sham group. Compared with PBS, ES-MSCs markedly suppressed the protein levels of cleaved caspase-3, Bax, and caspase-3 and promoted the protein level of Bcl-2 (Fig. 5b). These findings suggested that ES-MSCs suppressed apoptosis in skeletal muscle cells after ACS.

ES-MSCs alleviate skeletal muscle fibrosis and promote the regeneration of muscle fibers and muscle function recovery after ACS

To investigate skeletal muscle fibrosis and regeneration 7 days after ACS, we stained TA muscle sections with H\&E and Masson trichome. Skeletal muscle fibrosis and regenerated muscle fibers were significantly increased in ACS rats compared to sham rats, and ES-MSCs effectively reduced skeletal muscle fibrosis after ACS (Fig. 6a) and increased the number and diameter of regenerated muscle fibers (Fig. 6b). In addition, there was also an increase in the hanging grid test time (Fig. 6f) and hindlimb grip strength (Fig. 6g).

\section{ES-MSCs promotes M2 macrophage polarization and regulate inflammation in skeletal muscle after ACS}

To track the distribution of ES-MSCs after intravenous injection, PKH26-labeled ES-MSCs were infused into rats after fasciotomy, and the distribution was confirmed by examining frozen tissue sections after 1 day, 3 days, 5 days, and 7 days. PKH26-labeled ES-MSCs were found in the lung and skeletal muscle tissue (Figure 7a), and we found that the majority of cells populated the lung, while a few cells populated the skeletal muscle on 1 day. We also found that the cells disappeared from skeletal muscle tissue on the 
third day and from lung tissue on the fifth day in our study. Western blotting demonstrated that the level of CD206 (a marker of M2 macrophages) increased after 1 day and peaked at 3 days, after which the expression of CD206 gradually declined, and treatment with ES-MSCs increased CD206 expression in skeletal muscle after ACS (Fig. 7b). Immunofluorescence staining of macrophages on Day 3 also confirmed the increased inflation of M2 macrophages in skeletal muscle (Fig. 7d). Furthermore, quantitative real-time PCR showed that ES-MSC treatment significantly decreased the mRNA expression of the proinflammatory cytokines TNF- $a$ and IL- 6 and increased the anti-inflammatory cytokine IL-10 (Fig. 7e) 3 days after ACS.

\section{In vivo depletion of macrophages abolishes the therapeutic effect of ES-MSCs}

It was determined that anti-inflammatory CD206+ (M2) macrophages were increased in the ES-MSC treatment group. LC was injected into ES-MSC-treated ACS rats to deplete M2-polarized macrophages, and LV was administered as a control (Fig. 2, Part 3). Immunohistochemistry showed a significant decrease in the macrophage marker CD68 in skeletal muscle tissue (Fig. 8a), and western blotting also confirmed a decrease in the macrophage marker CD68 and M2 macrophage marker CD206 in skeletal muscle tissue 3 days after macrophage depletion (Fig. 8b). Skeletal muscle fiber injury and serum CK levels showed that the depletion of M2-polarized macrophages could enhance skeletal muscle injury 3 days after ACS and ES-MSC treatment, and quantitative real-time PCR showed that the inflammatory response was further increased in skeletal muscle tissue (Fig. 8h).

\section{Discussion}

In the present study, we investigate the efficacy and underlying mechanism of ES-MSCs in ACS-induced skeletal muscle injury. It is shown that ES-MSC therapy may serve as an effective modality for ACS treatment, and the protective effects were related to the M2 macrophage polarization (Fig. 9). Those findings are based on the following evidence: 1) the administration of ES-MSCs significantly alleviates ACS-induced skeletal muscle injury; 2) infused ES-MSCs increase M2 macrophage infiltration and regulate the inflammatory response in injured skeletal muscle after ACS; 3 ) the beneficial effects of ESMSCs were abolished by the depletion of macrophages.

Experimental evidence suggests that inflammation is one of the main driving factors of secondary injury following ACS [32-34]. Recent studies have shown that transplanted MSCs reduce IRI in skeletal muscle by inhibiting the inflammatory response and oxidative stress $[35,36]$ while promoting muscle tissue repair after injury [37]. Similar to previous studies that reported beneficial effects of other MSCs on skeletal muscle injury, ES-MSC therapy can effectively reduce the inflammatory response in vivo and inflammation-induced skeletal muscle edema 1 day after ACS, and the decrease in serum CK levels suggests that ES-MSCs reduce skeletal muscle cell injury after ACS. Histological analysis of the TA muscle on Day 1 also confirmed that ES-MSCs significantly reduced inflammatory cell infiltration and the disorganization of skeletal muscle fibers. Apoptosis is considered to be an inevitable type of cell death 
induced by IRI [38-40], especially in skeletal muscle [41, 42]. Because muscle edema began to resolve on Day 3 in our study, we evaluated the level of apoptosis in TA muscle tissue as an indicator of muscle injury after ACS. By decreasing inflammation, ES-MSCs significantly attenuated apoptosis and regulated the expression of apoptosis-associated proteins. These findings suggest that ES-MSCs can reduce skeletal muscle injury and subsequent apoptosis by reducing inflammation after ACS. Regeneration and fibrosis in injured skeletal muscle usually occur between 7 and 10 days after injury [27]. We chose 7 days after ACS to evaluate the effect of ES-MSCs on the regeneration and fibrosis of skeletal muscle injury. Histological analysis showed that ES-MSCs significantly decreased fibrosis and enhanced muscle regeneration (both myofiber numbers and diameters) 7 days after ACS injury. With the observed increase in muscle function recovery, our data suggest that ES-MSCs can reduce fibrosis, enhance skeletal muscle regeneration, and improve functional recovery of skeletal muscle after ACS-induced injury. These data suggest that ES-MSC therapy can ameliorate skeletal muscle injury induced by ACS, but the exact mechanism remains unknown.

Macrophages are the main source of cytokines, chemokines and growth factors that guide inflammation and repair after skeletal muscle injury [43], and exhibit two phenotypes with different functions: the classically activated subtype (M1) displays a proinflammatory profile, and the alternatively activated subtype (M2) exhibits anti-inflammatory and tissue repair properties [44]. Previous studies have shown that other sources of MSCs have beneficial effects on IRI-related injury by inducing M2 macrophage polarization [45-47], and experiments showed that macrophages cocultured with MSCs acquired an M2 phenotype [48]. However, the role of M2 macrophages in ES-MSCs treated IRI-related skeletal muscle injury, such as that in ACS, remains unclear. Therefore, we labeled ES-MSCs before injection and investigated the relationship between ES-MSC therapy and M2 macrophages in ACS injury. ES-MSCs disappeared from skeletal muscle tissue on the third day, suggesting that other cells may play a role in ES-MSC therapy. As a cell membrane protein, CD206 expression is one characteristic of M2 macrophages [49]. Western blotting indicated that ES-MSC therapy increased the expression of CD206.

Immunofluorescence staining further confirmed that M2 macrophage infiltration was increased in skeletal muscle tissue in the ES-MSC treatment group. The mRNA expression of inflammatory factors was analyzed, and the results showed that inflammation was reduced in the cell therapy group 3 days after ACS, at which time M2 macrophage infiltration in skeletal muscle was the most abundant.

M2 macrophages are involved in inhibiting inflammation and initiating the repair process in damaged muscle tissue $[44,50]$. To confirm the role of M2 macrophages in ES-MSCs mediated attenuation of ACSinduced skeletal muscle injury, we established a rat model of macrophage depletion by intravenous injection of LC as previously described [22]. Because the infiltration of M2 macrophages was most abundant on the third day in our study, we selected 3 days after ACS as the observation point. Our results showed that the protective effect of ES-MSCs on ACS-induced skeletal muscle injury was weakened after macrophage depletion, and inflammation in skeletal muscle was further increased. These observations suggest that ES-MSC treatment plays a protective role in skeletal muscle through M2 macrophages. 
This study was the first to demonstrate an improvement in ACS-induced skeletal muscle injury mediated by human ES-MSCs. Our study identified a stable, reliable, and consistent source of MSCs for treating ACS or similar skeletal muscle IRI diseases in the clinic. Furthermore, our study of the roles of M2 macrophages in ES-MSC-mediated treatment of ACS-induced skeletal muscle injury should provide the basis for the functional studies needed to determine the efficacy of ES-MSCs in the future.

Several limitations should not be ignored in the current study. First, a single dose of ES-MSCs was chosen to treat the animals in this study, the optimal dosage of ES-MSCs needs to be confirmed in the future.

Second, although the mechanism by which other sources of MSCs regulate macrophage polarization has been widely studied, the exact interaction between ES-MSCs and macrophage polarization under ACS conditions has not been studied. Third, the findings of a rodent study may not necessarily apply to humans, and macrophages may have differences between humans and rats. Therefore, further studies on clinical applications and elucidating the role of ES-MSCs in regulating macrophage polarization would be desirable.

\section{Conclusions}

Our findings suggest that embryonic stem cell-derived mesenchymal stem cells infusion could effectively alleviate ACS-induced skeletal muscle injury, in which the protective effects were related to M2 macrophage polarization.

\section{Abbreviations}

ACS Acute compartment syndrome

IRI Ischemia-reperfusion injury;

MSCs Mesenchymal stem cells;

ES Embryonic stem cell;

ES-MSCs Embryonic stem cell-derived mesenchymal stem cells;

EBs Embryoid bodies;

TA Tibialis anterior;

CK Creatine kinase;

ELISA Enzyme-linked immunosorbent assay;

TUNEL Terminal dUTP nick end labeling;

LC Liposomal clodronate; 


\section{Declarations}

\section{Acknowledgments}

The authors thank Dr. Jun Wei, Xue Wang, and Zhehou Cao for their contribution to the preparation of ESMSCs.

\section{Authors' contributions}

MZ designed the study. XJ and JY performed the experiments. XJ, FL, JYand MZ provided the materials. $\mathrm{XJ}$ and $\mathrm{JY}$ performed the measurements and analyzed the data. XJ, JY, FL, and JX wrote the manuscript. All authors contributed to the article and approved the submitted version.

\section{Fundings}

This study was supported by the Natural Science Foundation of China (82072126), the Zhejiang Provincial Key Research and Development Program of China (2021C03073, 2021C03036),the Key Program Cosponsored by Zhejiang Province and National Health Commission of China (WKJ-ZJ-2207).

\section{Availability of data and materials}

The original contributions presented in the study are included in the article. Further inquiries can be available on request to the corresponding author.

\section{Ethics approval and consent to participate}

All animal experiments were approved by the Institutional Ethics Committee of the Second Affiliated Hospital of Zhejiang University School of Medicine (Approval Number: [2021]049).Methods for each procedure were performed in accordancewith the approved guidelines and regulations.

\section{Consent for publication}

Not applicable.

\section{Competing interests}

The authors have declared that no competing interest exists.

\section{Footnotes}

Springer Nature remains neutral with regard to jurisdictional claims in published maps and institutional affiliations.

$\mathrm{XJ}$ and $\mathrm{JY}$ contributed equally to this work. 


\section{References}

1. von Keudell AG, Weaver MJ, Appleton PT, Bae DS, Dyer GSM, Heng M, et al. Diagnosis and treatment of acute extremity compartment syndrome. The Lancet. 2015;386(10000):1299-310.doi:

10.1016/S0140-6736(15)00277-9

2. Osborn PM, Schmidt AH. Diagnosis and Management of Acute Compartment Syndrome. The Journal of the American Academy of Orthopaedic Surgeons. 2021;29(5):183-8. doi: $10.1002 /$ mus. 24461

3. Pottecher J, Lefort H, Adam P, Barbier O, Bouzat P, Charbit J, et al. Guidelines for the acute care of severe limb trauma patients. Anaesth Crit Care Pain Med. 2021;40(4):100862.doi:

10.1097/MEJ.0000000000000835

4. Zibis A, Varitimidis S, Fyllos A, Raoulis V, Karachalios T, Malizos K. An observational study of complications in patients with established multiple compartments syndrome of the leg. Archives of orthopaedic and trauma surgery. 2021;141(2):253-9. doi: 10.1007/s00402-020-03488-2

5. Du W, Hu X, Shen Y, Teng X. Surgical management of acute compartment syndrome and sequential complications. BMC musculoskeletal disorders. 2019;20(1):98. doi: 10.1186/s12891-019-2476-5

6. Mithoefer K, Lhowe DW, Vrahas MS, Altman DT, Erens V, Altman GT. Functional outcome after acute compartment syndrome of the thigh. J Bone Joint Surg Am. 2006;88(4):729-37. doi:

10.2106/JBJS.E.00336

7. Chiu CH, Chang TH, Chang SS, Chang GJ, Chen AC, Cheng CY, et al. Application of Bone MarrowDerived Mesenchymal Stem Cells for Muscle Healing After Contusion Injury in Mice. Am J Sports Med. 2020;48(5):1226-35. doi: 10.1177/0363546520905853

8. Su WH, Wang CJ, Fu HC, Sheng CM, Tsai CC, Cheng JH, et al. Human Umbilical Cord Mesenchymal Stem Cells Extricate Bupivacaine-Impaired Skeletal Muscle Function via Mitigating NeutrophilMediated Acute Inflammation and Protecting against Fibrosis. International journal of molecular sciences. 2019;20(17). doi: 10.3390/ijms20174312

9. Arthur A, Gronthos S. Clinical Application of Bone Marrow Mesenchymal Stem/Stromal Cells to Repair Skeletal Tissue. International journal of molecular sciences. 2020;21(24).doi: 10.3390/ijms21249759

10. Helal MAM, Shaheen NEM, Abu Zahra FA. Immunomodulatory capacity of the local mesenchymal stem cells transplantation after severe skeletal muscle injury in female rats. Immunopharmacology and immunotoxicology. 2016;38(6):414-22. doi: 10.1080/08923973.2016.1222617

11. Lukomska B, Stanaszek L, Zuba-Surma E, Legosz P, Sarzynska S, Drela K. Challenges and Controversies in Human Mesenchymal Stem Cell Therapy. Stem Cells Int. 2019;2019:9628536. doi: $10.1155 / 2019 / 9628536$

12. Kozlowska U, Krawczenko A, Futoma K, Jurek T, Rorat M, Patrzalek D, et al. Similarities and differences between mesenchymal stem/progenitor cells derived from various human tissues. World J Stem Cells. 2019;11(6):347-74. doi: 10.4252/wjsc.v11.i6.347 
13. Uder C, Brückner S, Winkler S, Tautenhahn HM, Christ B. Mammalian MSC from selected species: Features and applications. Cytometry A. 2018;93(1):32-49. doi: 10.1002/cyto.a.23239

14. Thomson JA, Itskovitz-Eldor J, Shapiro SS, Waknitz MA, Swiergiel JJ, Marshall VS, et al. Embryonic stem cell lines derived from human blastocysts. Science. 1998;282(5391):1145-7. doi: $10.1126 /$ science.282.5391.1145

15. Xing D, Wang K, Wu J, Zhao Y, Liu W, Li JJ, et al. Clinical-Grade Human Embryonic Stem Cell-Derived Mesenchymal Stromal Cells Ameliorate the Progression of Osteoarthritis in a Rat Model. Molecules. 2021;26(3). doi: 10.3390/molecules26030604

16. Li E, Zhang Z, Jiang B, Yan L, Park JW, Xu RH. Generation of Mesenchymal Stem Cells from Human Embryonic Stem Cells in a Complete Serum-free Condition. International journal of biological sciences. 2018;14(13):1901-9. doi: 10.7150/ijbs.25306

17. Lee EJ, Lee HN, Kang HJ, Kim KH, Hur J, Cho HJ, et al. Novel embryoid body-based method to derive mesenchymal stem cells from human embryonic stem cells. Tissue Eng Part A. 2010;16(2):705-15. doi: 10.1089/ten.tea.2008.0596

18. Hawkins KE, Corcelli M, Dowding K, Ranzoni AM, Vlahova F, Hau KL, et al. Embryonic Stem CellDerived Mesenchymal Stem Cells (MSCs) Have a Superior Neuroprotective Capacity Over Fetal MSCs in the Hypoxic-Ischemic Mouse Brain. Stem Cells Transl Med. 2018;7(5):439-49. doi: 10.1002/sctm.17-0260

19. Wang X, Kimbrel EA, ljichi K, Paul D, Lazorchak AS, Chu J, et al. Human ESC-derived MSCs outperform bone marrow MSCs in the treatment of an EAE model of multiple sclerosis. Stem Cell Reports. 2014;3(1):115-30. doi: 10.1016/j.stemcr.2014.04.020

20. Lawendy AR, Sanders DW, Bihari A, Parry N, Gray D, Badhwar A. Compartment syndrome-induced microvascular dysfunction: an experimental rodent model. Can J Surg. 2011;54(3):194-200. doi: $10.1503 /$ cjs. 048309

21. Lawendy AR, Bihari A, Sanders DW, McGarr G, Badhwar A, Cepinskas G. Contribution of inflammation to cellular injury in compartment syndrome in an experimental rodent model. The bone \& joint journal. 2015;97-b(4):539-43.doi: 10.1302/0301-620X.97B4.34965

22. de Couto G, Liu W, Tseliou E, Sun B, Makkar N, Kanazawa H, et al. Macrophages mediate cardioprotective cellular postconditioning in acute myocardial infarction. The Journal of clinical investigation. 2015;125(8):3147-62. doi: 10.1172/JCl81321

23. Song M, Chen FF, Li YH, Zhang L, Wang F, Qin RR, et al. Trimetazidine restores the positive adaptation to exercise training by mitigating statin-induced skeletal muscle injury. J Cachexia Sarcopenia Muscle. 2018;9(1):106-18. doi: 10.1002/jcsm.12250

24. Xue QL, Yang H, Li HF, Abadir PM, Burks TN, Koch LG, et al. Rapamycin increases grip strength and attenuates age-related decline in maximal running distance in old low capacity runner rats. Aging. 2016;8(4):769-76. doi: 10.18632/aging.100929

25. Erkanli K, Kayalar N, Erkanli G, Ercan F, Sener G, Kirali K. Melatonin protects against ischemia/reperfusion injury in skeletal muscle. Journal of pineal research. 2005;39(3):238-42. doi: 
10.1111/j.1600-079X.2005.00240.x

26. Hurme T, Kalimo H. Activation of myogenic precursor cells after muscle injury. Medicine and science in sports and exercise. 1992;24(2):197-205.

27. Ota S, Uehara K, Nozaki M, Kobayashi T, Terada S, Tobita K, et al. Intramuscular transplantation of muscle-derived stem cells accelerates skeletal muscle healing after contusion injury via enhancement of angiogenesis. Am J Sports Med. 2011;39(9):1912-22. doi:

$10.1177 / 0363546511415239$

28. McCormack MC, Kwon E, Eberlin KR, Randolph M, Friend DS, Thomas AC, et al. Development of reproducible histologic injury severity scores: skeletal muscle reperfusion injury. Surgery. 2008;143(1):126-33. doi: 10.1016/j.surg.2007.06.005

29. Nie M, Liu J, Yang Q, Seok HY, Hu X, Deng ZL, et al. MicroRNA-155 facilitates skeletal muscle regeneration by balancing pro- and anti-inflammatory macrophages. Cell death \& disease. 2016;7(6):e2261. doi: 10.1038/cddis.2016.165

30. Ma W, Zhang R, Huang Z, Zhang Q, Xie X, Yang X, et al. PQQ ameliorates skeletal muscle atrophy, mitophagy and fiber type transition induced by denervation via inhibition of the inflammatory signaling pathways. Ann Transl Med. 2019;7(18):440. doi: 10.21037/atm.2019.08.101

31. Souza NHC, Mesquita-Ferrari RA, Rodrigues M, da Silva DFT, Ribeiro BG, Alves AN, et al. Photobiomodulation and different macrophages phenotypes during muscle tissue repair. J Cell Mol Med. 2018;22(10):4922-34. doi: 10.1111/jcmm.13757

32. Oyster N, Witt M, Gharaibeh B, Poddar M, Schneppendahl J, Huard J. Characterization of a compartment syndrome-like injury model. Muscle Nerve. 2015;51(5):750-8.doi: 10.1002/mus.24461. Epub 2015 Mar 31

33. Bihari A, Cepinskas G, Sanders D, Lawendy AR. Systemic Administration of Carbon MonoxideReleasing Molecule-3 Protects the Skeletal Muscle in Porcine Model of Compartment Syndrome. Crit Care Med. 2018;46(5):e469-e72.doi: 10.1097/CCM.0000000000002998

34. Bihari A, Chung KA, Cepinskas G, Sanders D, Schemitsch E, Lawendy AR. Carbon monoxide-releasing molecule-3 (CORM-3) offers protection in an in vitro model of compartment syndrome. Microcirculation. 2019;26(7):e12577.doi: 10.1111/micc.12577

35. Yin TC, Wu RW, Sheu JJ, Sung PH, Chen KH, Chiang JY, et al. Combined Therapy with Extracorporeal Shock Wave and Adipose-Derived Mesenchymal Stem Cells Remarkably Improved Acute IschemiaReperfusion Injury of Quadriceps Muscle. Oxid Med Cell Longev. 2018;2018:6012636.doi: $10.1155 / 2018 / 6012636$

36. Koch JM, D'Souza SS, Schwahn DJ, Dixon I, Hacker TA. Mesenchymoangioblast-derived mesenchymal stromal cells inhibit cell damage, tissue damage and improve peripheral blood flow following hindlimb ischemic injury in mice. Cytotherapy. 2016;18(2):219-28.doi:

10.1016/j.jcyt.2015.10.013

37. Ruehle MA, Stevens HY, Beedle AM, Guldberg RE, Call JA. Aggregate mesenchymal stem cell delivery ameliorates the regenerative niche for muscle repair. J Tissue Eng Regen Med. 2018;12(8):1867- 
76.doi: $10.1002 /$ term.2707

38. Heusch G. Myocardial ischaemia-reperfusion injury and cardioprotection in perspective. Nature reviews Cardiology. 2020;17(12):773-89.doi: 10.1038/s41569-020-0403-y

39. Wetzel MD, Wenke JC. Mechanisms by which hydrogen sulfide attenuates muscle function following ischemia-reperfusion injury: effects on Akt signaling, mitochondrial function, and apoptosis. J Transl Med. 2019;17(1):33.doi: 10.1186/s12967-018-1753-7

40. Wu MY, Yiang GT, Liao WT, Tsai AP, Cheng YL, Cheng PW, et al. Current Mechanistic Concepts in Ischemia and Reperfusion Injury. Cell Physiol Biochem. 2018;46(4):1650-67.doi: 10.1186/s12967018-1753-7

41. Zhang J, Zheng X, Wang P, Wang J, Ding W. Role of apoptosis repressor with caspase recruitment domain (ARC) in cell death and cardiovascular disease. Apoptosis. 2021;26(1-2):24-37.doi: 10.1007/s10495-020-01653-x

42. Lintz JA, Dalio MB, Tirapelli LF, Ribeiro MS, Joviliano EE, Piccinato CE. Effects of Postconditioning on Skeletal Muscle Injury and Apoptosis Induced by Partial Ischemia and Reperfusion in Rats. Annals of vascular surgery. 2017;40:285-93.doi: 10.1016/j.avsg.2016.10.032

43. Chazaud B. Inflammation and Skeletal Muscle Regeneration: Leave It to the Macrophages! Trends Immunol. 2020;41(6):481-92.doi: 10.1016/j.it.2020.04.006

44. Wu Y, Hirschi KK. Tissue-Resident Macrophage Development and Function. Front Cell Dev Biol. 2020;8:617879.doi: 10.1016/j.immuni.2016.02.024

45. Zhang Z, Tian H, Yang C, Liu J, Zhang H, Wang J, et al. Mesenchymal Stem Cells Promote the Resolution of Cardiac Inflammation After Ischemia Reperfusion Via Enhancing Efferocytosis of Neutrophils. Journal of the American Heart Association. 2020;9(5):e014397.doi:

10.1161/JAHA.119.014397

46. Zhao J, Li X, Hu J, Chen F, Qiao S, Sun X, et al. Mesenchymal stromal cell-derived exosomes attenuate myocardial ischaemia-reperfusion injury through miR-182-regulated macrophage polarization. Cardiovasc Res. 2019;115(7):1205-16.doi: 10.1093/cvr/cvz040

47. Wise AF, Williams TM, Kiewiet MB, Payne NL, Siatskas C, Samuel CS, et al. Human mesenchymal stem cells alter macrophage phenotype and promote regeneration via homing to the kidney following ischemia-reperfusion injury. Am J Physiol Renal Physiol. 2014;306(10):F1222-35.doi: 10.1152/ajprenal.00675.2013

48. Geng Y, Zhang L, Fu B, Zhang J, Hong Q, Hu J, et al. Mesenchymal stem cells ameliorate rhabdomyolysis-induced acute kidney injury via the activation of M2 macrophages. Stem cell research \& therapy. 2014;5(3):80.doi: 10.1186/scrt469

49. Perdiguero EG, Geissmann F. The development and maintenance of resident macrophages. Nat Immunol. 2016;17(1):2-8.doi: 10.1038/ni.3341

50. Vannella KM, Wynn TA. Mechanisms of Organ Injury and Repair by Macrophages. Annual review of physiology. 2017;79:593-617.doi: 10.1146/annurev-physiol-022516-034356 


\section{Figures}

\section{Figure 1}

Acquisition and identification of ES-MSCs. a Representative morphology of ES-MSCs at different stages.bThe expression of MSC-specific surface markers was analyzedby flow cytometry. Scale bar=300 $\mu \mathrm{m}$

\section{Figure 2}

Experimental design and animal groups

\section{Figure 3}

Effects of ES-MSCs on muscle edema and serum creatine kinase (CK) levels after ACS. aMuscle edema index 1 day, 3 days, and 7 days after ACS.b Serum CK levels 1 day, 3 days, and 7 days after ACS. N=5. The data are presented as the mean $\pm S D$, and there were no significant differences among the sham groups. ${ }^{*} P<0.05$ versus sham, ${ }^{\#} P<0.05$ versus ACS treated with PBS at the corresponding times.

\section{Figure 4}

Effect of ES-MSCs on systemic inflammation and the histopathology of skeletal muscle 1 day after ACS. a-cTheserum levels of the cytokines TNF- $a$, IL-6, and IL-10 were measured by ELISA. $\mathbf{d}$ Skeletal muscle damage scores 1 day after ACS. $\mathrm{N}=5$. e-h Representative images of the TA muscle cross sections in each group (original magnification, 200x). The data are presented as the mean $\pm S D$, and there were no significant differences among the sham groups. ${ }^{*} P<0.05$ versus sham, ${ }^{\sharp} P<0.05$ versus ACS treated with PBS.

\section{Figure 5}

Effect of ES-MSCs on skeletal muscle apoptosis 3 days after ACS. a Representative images of TUNEL and dystrophin staining. b Representative western blot images. c Quantitative analysis of TUNEL and dystrophin staining. $\mathbf{d}$ Quantitative analysis of cleaved caspase3 expression. e Quantitative analysis of 
Bax expression. $\mathbf{f}$ Quantitative analysis of Bcl-2 expression. $\mathbf{g}$ Quantitative analysis of cleaved caspase3 expression. $\mathrm{N}=4$. The data are presented as the mean $\pm S D$, and there were no significant differences among the sham groups. ${ }^{*} P<0.05$ versus sham, ${ }^{\#} P<0.05$ versus ACS treated with PBS.

\section{Figure 6}

Effect of ES-MSCs on skeletal muscle fibrosis, regeneration, and functional recovery 7 days after ACS. aRepresentative imagesof fibrosis in each group,as shown by Masson trichrome staining. $\mathbf{b}$ Representative images of muscle regeneration, as shown by H\&E staining. Regenerating myofibers were defined by myofibers containing a central nucleus (original magnification, 200x). c Quantitative analysis of the fibrotic area in each group. $\mathbf{d}$ and $\mathbf{e}$ Quantitative analysis of the number (d) and diameter (e) of regenerating myofibers in each group. $f$ and gThe grip strength (f) and hanging time (g) in each group. $N=5$. The data are presented as the mean $\pm S D$, and there were no significant among the sham groups. ${ }^{*} P<0.05$ versus sham, ${ }^{\#} P<0.05$ versus ACS treated with PBS.

\section{Figure 7}

Effects of ES-MSCs on macrophage polarization and the mRNA expression of inflammatory factors in skeletal muscle after ACS. aThe distribution of labeled ES-MSCs in the lung and skeletal muscle tissue of rats at various time points. b Representative western blot images. c Quantitative analysis of CD206 expression. $\mathbf{d}$ Representative images of macrophage (CD68) and M2-polarized macrophage (CD206) infiltration in skeletal muscle after PBSor ES-MSC infusion.e Quantitative real-time PCR data showing the mRNA levels of TNF-a, IL-6, and IL-10 in skeletal muscle after PBS or ES-MSC infusion. $N=4$. The data are presented as the mean \pm SD. ${ }^{*} P<0.05$ versus ACS treated with PBS.

\section{Figure 8}

Depletion of macrophages using LC abolishes the therapeutic effect of ES-MSCs. a Immunohistochemical evaluation of CD68+ macrophage infiltration after macrophage depletion.b Representative images showing CD68 and CD206 expression in skeletal muscle after macrophage depletion. cQuantitative analysis of CD68 expression. $\mathbf{d}$ Quantitative analysis of CD206 expression.e Representative images of skeletal muscle stained with H\&E after macrophage depletion. $\mathbf{f}$ Quantitative analysis of skeletal muscle injury scores after macrophage depletion. $\mathbf{g}$ Serum CK levels after macrophage depletion. $N=5$. The data are presented as the mean \pm SD. $* P<0.05$ versus rats injected with LV. $\mathbf{h}$ Quantitative real-time PCR data showing the mRNA levels of TNF-a, IL-6, and IL-10 in skeletal 
muscle after macrophage depletion. $\mathrm{N}=4$. The data are presented as the mean $\pm \mathrm{SD}$. ${ }^{\star} P<0.05$ versus rats injected with LV.

\section{Figure 9}

Schematic mechanism by which ES-MSCs ameliorate skeletal muscle injury induced by ACS. 